\title{
Correction to: Quality of life of survivors 1 year after the diagnosis of diffuse large B-cell lymphoma: a LYSA study
}

\author{
Alexandra-Cristina Paunescu ${ }^{1}$. Christiane Copie Bergman ${ }^{2} \cdot$ Sandra Malak $^{3}$. Steven Le Gouill ${ }^{4}$ Vincent Ribrag $^{5}$. \\ Krimo Bouabdallah ${ }^{6,7}$. David Sibon ${ }^{8}$. Gerhard Rumpold ${ }^{9} \cdot$ Marie Preau $^{10} \cdot$ Nicolas Mounier $^{11}$. Corinne Haioun ${ }^{12}$. \\ Fabrice Jardin ${ }^{13} \cdot$ Caroline Besson ${ }^{1,14}$
}

Published online: 25 October 2021

๑) Springer-Verlag GmbH Germany, part of Springer Nature 2021

\section{Correction to: Annals of Hematology https://doi.org/10.1007/s00277-021-04689-4}

In the version of this article initially published, the last names and first names of all the authors were inverted.

The original article has been corrected.

Publisher's note Springer Nature remains neutral with regard to jurisdictional claims in published maps and institutional affiliations.

The original article can be found online at https://doi.org/10.1007/ s00277-021-04689-4.

Caroline Besson cbesson@ch-versailles.fr

Alexandra-Cristina Paunescu alexandra-cristina.paunescu@gustaveroussy.fr

1 Inserm CESP U1018, Gustave Roussy, 114 Rue Edouard Vaillant, 94805 Villejuif, France

2 Department of Pathology, CHU Henri Mondor, AP-HP Paris, France

3 Hematology, Curie, Saint-Cloud, France

4 Service d'hematologie clinique du CHU de Nantes, INSERM CRCINA Nantes-Angers, NeXT Universite de Nantes, Nantes, France

5 DITEP and Hematology department, Gustave Roussy, Villejuif, France

6 Department Hematology, University Hospital of Bordeaux, Pessac, France
7 Hopitaux de Bordeaux CHU, Bordeaux, France

8 INSERM UMR 1163 \& CNRS URL 8254, Hematology Department, Necker University Hospital, APHP, Paris, France

9 Department of Medical Psychology, Medical University Innsbruck, Innsbruck, Austria

10 UR GRePS, Université Lumière Lyon 2, Institut de Psychologie, Bron, France

11 Department of Onco-Hematology, Archet Hospital, Nice, France

12 Lymphoid Malignancies Unit, Henri Mondor University Hospital, AP-HP, UPEC, Creteil, France

13 Department of Hematology, Centre Henri Becquerel, Rouen, France

14 Centre Hospitalier de Versailles, Hopital Andre Mignot, Le Chesnay, 177 Rue de Versailles, 78157 Versailles, France 